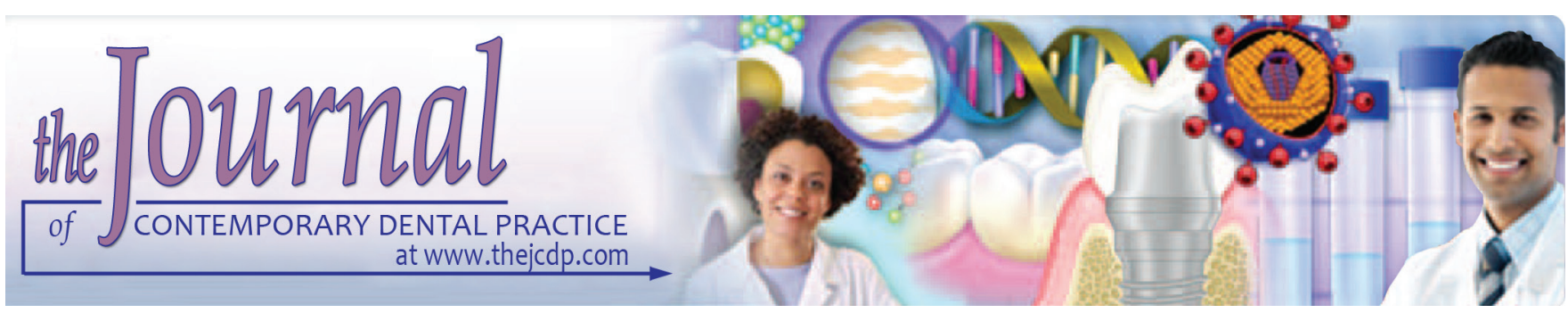

\title{
Prevalence of Candida spp. in Healthy Oral Mucosa Surfaces with Higher Incidence of Chronic Hyperplastic Candidosis
}

${ }^{1}$ Claúdia Arruda, ${ }^{2}$ Gabriela Artico, ${ }^{3}$ Roseli Freitas, ${ }^{4}$ Antônio Filho, ${ }^{5}$ Dante Migliari

\begin{abstract}
Introduction: Predisposing factors in chronic hyperplastic candidosis $(\mathrm{CHC})$ have been poorly recognized. This study aimed at assessing the prevalence of Candida spp. in areas of the oral mucosa showing greater prevalent rate of $\mathrm{CHC}$, such as the retrocomissural area, the lateral borders of the tongue, and the hard-palate mucosa in four groups of individuals presenting predisposing factors as follows: Smoking habits (group I); patients with low salivary flow rate (SFR) (hyposalivation - group II); patients with loss of vertical dimension of occlusion (LVDO group III); and control subjects (group IV).
\end{abstract}

Materials and methods: A total of 44 individuals (age 40 90 years, mean: 55.8 years) were divided into four groups: Group I (11 smokers); group II (10 hyposalivation patients); group III (10 LVDO patients); and group IV (control, 13 healthy subjects). All individuals were tested for Candida-pseudohyphae form by direct examination and for Candida spp. culture growth in samples obtained from the retrocomissural, tongue's lateral border, and hard-soft palatal mucosa.

Results: Direct examination showed a statistically significant prevalence rate for pseudohyphae $(p<0.05)$ on the retrocomissural and on tongue's lateral borders of individuals with LVDO. A statistically significant $(p<0.05)$ culture growth for Candida spp. was found on the retrocomissural areas of those with hyposalivation and with LVDO, and on the palate mucosa and on the tongue's lateral borders in the smokers and in the individuals with LVDO when compared with those of the control group.

Conclusion: While direct examination is effective for detecting pseudohyphae, LVDO and tobacco smoking seem to be factors of relevance to the development of $\mathrm{CHC}$.

1,2,5 Department of Stomatology, School of Dentistry, University of São Paulo, São Paulo, Brazil

${ }^{3,4}$ Laboratory of Medical Investigation, Division of Dermatology School of Medicine, University of São Paulo, São Paulo, Brazil

Corresponding Author: Dante Migliari, Associate Professor Disciplina de Estomatologia Clínica, Faculdade de Odontologia Universidade de São Paulo, São Paulo, Brazil, Phone: +551130917883, e-mail: damiglia@usp.br
Clinical significance: Since $\mathrm{CHC}$ has been linked to a high rate of malignant transformation, this study analyzes some clinical (and exogenous) factors that may contribute to the development of $\mathrm{CHC}$ and addresses some preventive measures to reduce its incidence.

Keywords: Candida spp., Chronic hyperplasic candidosis, Direct examination, Pseudohyphae.

How to cite this article: Arruda C, Artico G, Freitas R, Filho A, Migliari D. Prevalence of Candida spp. in Healthy Oral Mucosa Surfaces with Higher Incidence of Chronic Hyperplastic Candidosis. J Contemp Dent Pract 2016;17(8):618-622.

Source of support: Nil

Conflict of interest: None

\section{INTRODUCTION}

Chronic hyperplastic candidosis (CHC) is a variant of oral candidosis exhibiting a clinical presentation quite similar to that of an idiopathic leukoplakia, i.e., showing a white plaque that cannot be removed by scraping. Chronic hyperplastic candidosis can also display a homogeneous or nonhomogeneous surface. The sites most affected are the retrocomissural, palatal mucosa, and the lateral surfaces of the tongue. ${ }^{1-4}$

The CHC can (and should) be differentiated from oral leukoplakia of idiopathic origin since the former usually shows a complete (or substantial) resolution following appropriate antifungal therapy. ${ }^{1,3,4}$ The foremost concern on $\mathrm{CHC}$ lesion rests on its high potential for malignant transformation. Despite the lack of epidemiological study on CHC with regard to its rate of malignant transformation, there are substantial clinical evidence showing that the potential for malignization of $\mathrm{CHC}$ is higher than that of oral leukoplakia. ${ }^{1,2,4-6}$

Unlike the other forms of oral candidosis, such as the pseudomembranous and erythematous, in which a 
predisposing factor, either local or systemic, is invariably linked to the presence of the oral lesion - the CHC form develops without any association with a (known) predisposing factor. Studies investigating predisposing factors associated with $\mathrm{CHC}$ have surfaced some evidence that local factors, such as tobacco smoking, hyposalivation, loss of vertical dimension, acting independently or in combination with each other, may play a role in the development of CHC.

This study aims at investigating the frequency of Candida spp. in sites showing higher incidence of CHC (such as the retrocomissural area, the lateral borders of the tongue, and the hard-palate mucosa) in 4 groups of patients: (Group I) patients with smoking habits; (group II) patients with low salivary flow rate (SFR); (group III) patients with loss of vertical dimension of occlusion (LVDO); and (group IV) control subjects without systemic conditions that could predispose to oral candidosis (diabetes, long-term corticotherapy, or antibiotic therapy, or taking immunosuppressive agents).

\section{MATERIALS AND METHODS}

The present study was carried out at the Dental and Medical Schools of the University of São Paulo (Brazil). All individuals taking part of this study were at least 40 years old. A total of 44 individuals were selected and divided into four groups: Group I (smokers), comprised 11 smokers subjects (4 females and 7 males; 42-71 years, mean 53 years); group II (low SFR), comprised 10 subjects ( 8 females and 2 males; 45-90 years, mean 56 years); group III comprised 10 subjects showing LVDO (6 females and 4 males; 42-74 years, mean 62 years); group IV (control), comprised 13 healthy subjects without any of the (presumably) CHC predisposing factors (8 females and 5 males; range $41-81$ years, mean 62 years).

\section{Criteria for Selection of Groups}

Subjects were selected whether they met these specific criteria: Group I, smokers with a chronic habit of smoking (10-20 cigarettes a day for 10 years or more), with normal SFR (>0.1 mL/minute), with no removable prosthetic and without LVDO; group II, patients with a low SFR $(\leq 0.1 \mathrm{~mL} /$ minute), no smokers and without a LVDO; group III, patients with a LVDO, with normal SFR and no smokers. These patients were selected exclusively on clinical findings indicating LVDO as follows: Absence of posterior occlusion, either from the upper or lower jaw, or both (at least for more than 5 years, and nonwearers of partial or total removable prosthesis); group IV, control subjects were selected among individuals who came to our clinic seeking dental treatment. Individuals asked to participate were no smokers, no xerostomic and without LVDO.

\section{Criteria for Exclusion of Groups}

Subjects were excluded if they were taking broadspectrum antibiotics, antifungal, immunosuppressive, or glucocorticoids agents for a long period of time, or recently (within 3 months before the start of this study) had metabolic and/or hematological diseases (mainly a recent history of anemia by iron deficiency), chronic alcohol abuse, or daily use of mouthwash.

\section{Investigation of the SFR (Hyposalivation)}

Salivary flow rate was determined among subjects complaining of xerostomia, and that had agreed to participate in the study. As widely known, xerostomia (a subjective sensation of oral dryness) is not necessarily associated with a low SFR, but those complaining of xerostomia make up a group of individuals in whom hyposalivation is more often investigated than in nonxerostomic subjects. Hyposalivation was evaluated based on the value of the SFR using unstimulated (resting) whole saliva. Whole saliva was collected over a period of 15 minutes, between 9 and 10 o'clock in the morning, after 2 hours of fasting. The cut-off value for hyposalivation was defined when the SFR was $\leq 1.5 \mathrm{~mL} /$ minute, according to Vitali et al. ${ }^{7}$

\section{Direct Examination for Candida spp.}

Samples were obtained (in all groups of individuals) by scraping the oral mucosa sites (the retrocomissural areas, the lateral borders of the tongue and the hard-palate mucosa) using a scalpel blade, number 15 . The material was smeared onto a microscope slide and covered with a drop of $10 \%$ potassium hydroxide. A coverglass was placed over the smear and pressed gently to remove air bubbles. The immersed material was left for 3 minutes for clearing the visual field and then taken to a microscopic for examination. A positive result for Candida spp. was considered with the presence of pseudohyphae and budding cells (Fig. 1).

\section{Collection of Samples for Candida spp. Culture and Isolation}

Simultaneously to direct examination procedure, a collection for Candida spp. culture was also made by scraping the same sites of the oral mucosa using a cytobrush ${ }^{\circledR}$ (Vagispec, Brazil). After collection, samples were transferred to individual tubes containing $5 \mathrm{~mL}$ of sterile saline $(0.9 \% \mathrm{NaCl})$ and transported to an insulated container at the Laboratory of Medical Investigation, School of Medicine, University of São Paulo, Brazil, where it was processed. 




Fig. 1: Direct examination showing pseudohyphae and a few budding cells

\section{Identification of Candida spp.}

At the laboratory, samples were centrifuged at 5,000 rpm for 10 minutes for concentration of the solution, and a volume of approximately $50 \mu \mathrm{L}$ was inoculated on Sabouraud's dextrose agar (SDA, DIFCO/USA) and incubated at $28^{\circ} \mathrm{C}$ for 24 to 48 hours. Positive cultures were placed on CHROMagar ${ }^{\circledR}$ Candida medium (CHROMagar, Paris, France) for macroscopic identification of Candida species by color of the colonies. Isolated yeasts were inoculated in SDA for subsequent presumptive identification of Candida species by phenotypic methods using morphological features and biochemical properties, as follows: Incubation on sterile bovine fetal serum at $37^{\circ} \mathrm{C}$ for 3 hours for germ-tube formation (germ-tube test); incubation on cornmeal-Tween 80 medium at $28^{\circ} \mathrm{C}$ for 5 days for production of hyphae, pseudohyphae and chlamydospores; and assimilation test (carbohydrate and nitrogen) for patterns of fermentation and assimilation of carbon or nitrogen. ${ }^{8}$

For presumptive discrimination between Candida albicans and Candida dubliniensis, isolates identified as
C. albicans were analyzed phenotypically, as follows: Incubation on hypertonic Sabouraud broth (Sabouraud dextrose broth with $6.5 \% \mathrm{NaCl}$ ) for 96 hours, which is restrictive to C. albicans growth; incubation on Staib agar at $30^{\circ} \mathrm{C}$ for up to 5 days for chlamydospore formation, which is selective for C. dubliniensis $5^{5}$; incubation at $45^{\circ} \mathrm{C}$ for 48 hours and assimilation test (xylose and trehalose), which is restrictive to C. albicans. ${ }^{9}$

\section{Statistical Analysis}

The results were analyzed using the Fisher's exact test with the aid of Epi Info software for Windows (version 3.4., Centers for Diseases Control, Atlanta, GA, USA). Statistical significance was set for $\mathrm{p}<0.05$.

\section{RESULTS}

Individuals enrolled in this study were made up of 18 males and 26 females; age 40 to 90 years, mean: 55.8 years. The results of direct examination for detecting the presence of pseudohyphae showed that there was a higher and statistically significant prevalence rate for pseudohyphae $(\mathrm{p}<0.05)$ on the retrocomissural and on tongue's lateral borders surface of individuals with LVDO as compared with those of the smokers, of the individuals with hyposalivation, and of the control group; no statistical difference was found for the hard-soft palatal mucosa using the same comparison pattern among the groups (Table 1).

Growth of Candida spp. from the oral mucosal samples inoculated on SDA media was seen in all sites of all four groups with the exception of the retrocomissural mucosa of the control group (Table 1). Statistically significant number of sites with positive culture for Candida spp. was found in the retrocomissural areas of those with hyposalivation and with LVDO when compared with the control group. For the palate mucosa and the tongue's lateral borders, a statistically significant positive growth for Candida spp. was only found in the smokers and in

Table 1: Direct and culture examinations of Candida spp. of samples collected from the oral mucosa of the four groups $(\mathrm{n}=44)$

\begin{tabular}{|c|c|c|c|c|c|c|c|c|}
\hline & \multicolumn{8}{|c|}{ Groups } \\
\hline & \multicolumn{2}{|c|}{ Smokers $(n=11)$} & \multicolumn{2}{|c|}{ Hyposalivation $(n=10)$} & \multicolumn{2}{|c|}{$\operatorname{LVDO}(n=10)$} & \multicolumn{2}{|c|}{ Control $(n=13)$} \\
\hline & $p(\%)$ & $N E(\%)$ & $p(\%)$ & $N E(\%)$ & $p(\%)$ & $N E(\%)$ & $p(\%)$ & $N E(\%)$ \\
\hline \multicolumn{9}{|l|}{ Direct exam } \\
\hline Retrocomissural mucosa & 0 & $11(100)$ & 0 & $10(100)$ & $4(40)^{\dagger}$ & $6(60)$ & 0 & $13(100)$ \\
\hline Hard/soft palate & $2(18.2)$ & 9 (81.8) & 0 & $10(100)$ & $2(20)$ & $8(80)$ & 0 & $13(100)$ \\
\hline Lateral surface of tongue & 0 & $11(100)$ & 0 & $10(100)$ & $4(40)^{\dagger}$ & $6(60)$ & 0 & $13(100)$ \\
\hline \multicolumn{9}{|l|}{ Culture exam } \\
\hline Retrocomissural mucosa & $3(27.3)$ & $8(72.7)$ & $4(40)$ & $6(60)^{\dagger}$ & $6(60)^{\dagger}$ & $4(40)$ & 0 & $13(100)$ \\
\hline Hard/soft palate & $6(54.5)^{\dagger}$ & $5(45.4)$ & $3(30)$ & $7(70)$ & $5(50)^{\dagger}$ & $5(50)$ & $1(7.7)$ & $12(92.3)$ \\
\hline Lateral surface of tongue & $6(54.5)^{\dagger}$ & $5(45.4)$ & $4(40)$ & $6(60)$ & $7(70)^{\dagger}$ & $3(30)$ & $1(7.7)$ & $12(92.3)$ \\
\hline
\end{tabular}

$\mathrm{N}$ : Number of patients; LVDO: Loss of vertical dimension of occlusion; P: Positive; NE: Negative; 't: Significant by Fisher's exact test (two-tailed) 
the individuals with LDVO as compared with the control group (Table 1). Candida albicans was the predominant species in all individuals showing positive growth for Candida spp. The other species isolated was Candida tropicalis, from the palatal mucosa of two subjects: One with hyposalivation and the other smoker. Colonization by C. dubliniensis was not seen in any of the subjects.

\section{DISCUSSION}

As previously stated, $\mathrm{CHC}$ differs from the other form of oral candidosis by carrying a significant risk of malignant transformation and by occurring without the existence of a (known) predisposing factor. ${ }^{1-6}$

Despite the growing interest in studying $\mathrm{CHC}$ (mainly because of its great risk of malignization), there has been basically only one publication containing a significant number of patients (53 cases) with $\mathrm{CHC}$ since this condition was first described, as a pathologic entity, by Cawson in $1966 .{ }^{4,10}$ In that series of $53 \mathrm{CHC}$ cases, ${ }^{4}$ the authors have associated $\mathrm{CHC}$ with either a heavy smoking habit or denture wearing, or both, as these two factors were found to be significantly greater in $\mathrm{CHC}$ patients than in two control groups; $\mathrm{CHC}$ lesions in these patients were found predominantly on the commissure (bilaterally), on the palate, and on the tongue. Some patients would also have multiple sites involved. These findings were in agreement with the results of another (smaller) series of 12 cases of patients with $\mathrm{CHC}$ carried out many years before. $^{3}$

The present study yielded data that were closely related with those of the aforementioned studies. ${ }^{3,4}$ The most important of them was the significant presence of pseudohyphae on direct examination in samples retrieved from the retrocomissural mucosae and from the lateral surfaces of the tongue in individuals with LVDO. These sites are regarded as the most affected by $\mathrm{CHC}$.

Culture analyses showed positive results for C. albicans in all but one site in the four groups. In this respect, statistically significant results for higher prevalence rate of Candida spp. growth were found only when sites of subjects with LVDO, or with smoking habit or with hyposalivation were compared to those of the control group (Table 1). These data mean that the local factors analyzed in this study (cigarette smoking, hyposalivation, and LVDO) are of relevance for promoting oral colonization of C. albicans and, possibly, for developing oral candidosis.

But culture examination arguably shows $C$. albicans in a nonpathogenic form (a yeast-like fungus seen in many healthy individuals) which are not capable by itself of invading the tissues (the oral mucosa). In this respect, direct examination is of greater significance since a positive result (and diagnostic for Candida) is determined by the presence of pseudohyphae; this form being implicated in the fungus capability of invading the epithelium. $^{3,6,10,11}$

Direct examination positivity was predominantly found on the retrocomissural mucosa (4 cases), on the lateral tongue (4 cases), and on the palatal mucosa (2 cases) of individuals with LVDO. Three of these individuals showed a concomitant involvement of sites positive for pseudohyphae. The close contact between the oral mucosal surfaces most likely accounted for the direct examination positivity in multiple sites in the same individual.

The main aspect one can draw from this and the other two available reports on the issue of CHC is that LVDO most likely play a fundamental role in the development of this condition since lesions have been found more significantly in those wearing full-denture for a very long time and in those with angular cheilitis. Most of these patients are more susceptible to presenting a LVDO and, eventually, favoring the development of CHC. A high consumption of cigarette smoking may also be an important factor with regarding to lesions on the hard-soft palatal mucosa. The combination of both factors, LDVO and smoking, may critically enhance the chance for the development of CHC.

It has been demonstrated that the invasion of hyphae in the epithelium triggers an inflammatory and epithelial hyperplasia reaction. ${ }^{1,3,6}$ These events may chronically develop into $\mathrm{CHC}$ although the exact etiopathogenic mechanism of this condition is unknown.

\section{CONCLUSION}

Despite the small number of subjects involved in this study, the results presented here may add some insight in the CHC development and (hopefully) some basic approach for its prevention. This would include the use of direct examination (or cytological smear stained with periodic acid-Schiff) in search for pseudohyphae in patients wearing full or partial removal prosthesis or in those with angular cheilitis or in patients with absence of the posterior teeth. In case of a positive test for pseudohyphae, a clinician should find a way to remove the cause (usually by prosthetic rehabilitation) along with a treatment with topical antifungal agent. Quitting smoking is one of the utmost measures for preventing oral diseases, mainly those with potential for malignization, and this most likely includes $\mathrm{CHC}$. 


\section{REFERENCES}

1. Sitheeque MA, Samaranayake LP. Chronic hyperplastic candidosis/Candidiasis (Candidal leukoplakia). Crit Oral Biol Med 2003 Apr;14(4):253-267.

2. Scully C, El-Kabir M, Samaranayake LP. Candida and oral candidosis: a review. Crit Rev Oral Biol Med 1994 Feb;5(2):125-157.

3. Cawson RA, Lehner T. Chronic hyperplastic candidiasiscandida leukoplakia. Br J Dermatol 1968 Jan;80(1):9-16.

4. Arendorf TM, Walker DM, Kingdom RJ, Roll JRS, Newcombe RG. Tobacco smoking and denture wearing in oral candidal leukoplakia. Br Dent J 1983 Nov;155(10):340-343.

5. Odel EW, Morgan PR. Candidosis. In: Biopsy pathology of the oral tissues. 1st ed. London: Chapaman \& Hall; 1998. p. 153.

6. Field EA, Field K, Martin MV. Does Candida have a role in oral epithelial neoplasia. J Med Vet Mycol 1989 May;27(5): 277-294.
7. Vitali C, Bombrdieri S, Jonsson R, Moutsopoulos HM, Alexaander EL, Carsons SE, Daniels TE, Fox PC, Fox RI, Kassan SS, et al. Classification criteria for Sjögren's syndrome: a revised version of the European criteria proposed by the American-European Consensus Group. Ann Rheum Dis 2002 Jun;61(6):554-558.

8. Alves SH, Milan EP, Sant'Ana PL, Oliveira LO, Santurio JM, Colombo AL. Hypertonic Sabouraud broth as a simple and powerful test for Candida dubliniensis screening. Diagn Microbiol Infect Dis 2002 May;43(1):85-86.

9. Campanha NH, Neppelenbroek KH, Spolidorio DMP, Spolidorio LC, Pavarina AC. Phenotypic methods and commercial systems for the discrimination between C. albicans and C. dubliniensis. Oral Dis 2005 Nov;11(6):392-398.

10. Cawson RA. Chronic oral candidiasis and leukoplakia. Oral Surg Oral Med Oral Path 1966 Nov;22(5):582-591.

11. Vuckovic N, Bokor-Bratic M, Vukovic D, Picuric I. Presence of Candida albicans in potentially malignant oral mucosal lesions. Arch Oncol 2004 Jan;12(1):51-54. 